\title{
Correlation Analysis between Air Pollutants and Meteorological Factors
}

\author{
Huan Dong, Chao Chen*, Lecheng Huang, Cunxin Han, Bin Zhao \\ School of Computer Science, Sichuan University of Science and Engineering, Zigong 643000, China \\ *Correspondence Author, chenchao@suse.edu.cn
}

\begin{abstract}
In order to explore the composite relationship between meteorological factors affecting air quality and pollutant concentration, the high-resolution air pollution reanalysis open data set of China from 2013 to 2018 is used to calculate, visually design and analyze the big data of environmental monitoring by using the Pearson correlation coefficient PCC of statistical method. The experimental results show that meteorological factors have a great impact on pollutants, temperature has a negative correlation with pollutants, air pressure has a positive correlation, humidity has no significant correlation, wind speed has an obvious negative correlation with six pollutants, and has a relatively small impact on pollutant diffusion.
\end{abstract}

Keywords: Environmental of Big Data, Visualization, Air quality index, Correlation coefficient.

\section{Introduction}

Urban air pollution is an important part of national environmental protection. With the acceleration of China's urbanization process, urban environmental protection plays an increasingly important role in national environmental protection. Air pollution prevention and control is not only a major livelihood issue, but also an important starting point for economic upgrading[1]. In 2021, the three-year action plan for the defense of the blue sky issued by China's State Council officially ended. The national air quality continued to improve, showing a steady trend towards the better. However, the effectiveness of atmospheric control is still unstable. Especially since autumn and winter, fog and haze have reappeared in some cities, and the increasingly prominent regional compound air pollution problem has been accumulated for a long time, The concentration of air pollutants is also affected by many elements such as region, economy and meteorology, and there is a large gap in distribution space in the same period $[2,3]$.

In recent years, scholars at home and abroad have carried out a large number of studies on air pollution. The studies of Tan Lina and Fang Xiaoting show that there is a high correlation between the distribution of air pollutants and ground meteorological factors, which is obviously related to seasonal changes. The air quality in summer is obviously better than that in winter[4,5]. Yang Liu and others analyzed the variation characteristics of air pollutant mass concentration in Chengdu from 2014 to 2016 and the impact of precipitation on it[6].

The air pollutants affecting air quality and meteorological factors are complex and changeable, and the composite relationship and correlation between them need to be further clarified. As a statistical calculation method, Pearson correlation coefficient (PCC) can quantitatively measure the correlation between variables[7]. Wang Geng and others calculated the change of pollution characteristics and the correlation with meteorological factors in Zhangqiu district by using Pearson correlation coefficient[8]. Liu Qing and others pointed out that the ozone concentration showed a negative correlation with other pollutants and was greatly affected by the topographic and geomorphic characteristics[9]. Liu Zihao and others analyzed the temporal and spatial changes of air pollution in Hefei and its correlation with meteorological factors, pointing out that air pollutants have a negative correlation with air temperature and sunshine, a positive correlation with air pressure, and no obvious correlation with humidity and wind speed[10]. Li Bo et al. Obtained through correlation analysis that there is a significant typical correlation between pollutant concentration and meteorological elements, and temperature, wind speed and relative humidity in meteorological elements have a significant impact on gaseous pollutants[11]. The research of the above scholars is limited by the limitations of data source monitoring region and time interval, incomplete pollution indicators, too small sample data and other factors. In this paper, the correlation calculation and statistical analysis of ambient air pollutants and meteorological factors in cities in China will be carried out, and the big data visualization tool will be used to visually display the data on temporal and spatial series, so as to further explore the concentration influence mechanism between different pollutants, so as to provide reference for the study of the influencing factors of air pollution indicators, It will facilitate the environmental management departments to carry out dynamic decision-making by using routine air quality monitoring data, and promote the research on global climate governance.

\section{Data Sources and Analysis Methods}

\subsection{Data Sources}

This experiment uses the high-resolution air pollution reanalysis open data set of China from 2013 to 2018, which is jointly issued by the Institute of Atmospheric Physics of the Chinese Academy of Sciences and other units, and provides a large number of air quality data with high-dimensional and time-series characteristics collected by the national air quality monitoring station[12].

\subsection{Data Preprocessing}

Air quality index (AQI) simplifies the monitored air concentration into a single conceptual index value form according to the proportion of various components in the air. 
It expresses the degree of air pollution and air quality by classification, which is suitable for expressing the short-term air quality status and change trend of cities.

In this experiment, the pollutants are measured uniformly according to the ambient air quality standard of the people's Republic of China. Combined with the air quality sub index in the standard and the corresponding pollutant project concentration limit, the air quality sub index (IAQI) is calculated respectively according to the measured concentration values of PM2.5, PM10, $\mathrm{SO}_{2}, \mathrm{NO}_{2}, \mathrm{CO}, \mathrm{O}_{3}$ and other pollutants. The calculation formula is as follows (1):

$$
I A Q I_{p}=\frac{I A Q I_{H i}-I A Q I_{L o}}{B P_{H i}-B P_{L o}}\left(C_{p}-B P_{L o}\right)+I A Q I_{L o}
$$

$I A Q I_{p}$ is Air quality sub index of pollutant project $P ; C_{p}$ is the mass concentration value of pollutant item $P ; B P_{H i}$ is the upper limit value of pollutant concentration; $B P_{L o}$ is the lower limit of pollutant concentratio; $I A Q I_{H i}$ is corresponding air quality sub index of $B P_{H i} ; I A Q I_{L o}$ is corresponding air quality sub index of $B P$;

The AQI of all districts and counties, the smallest administrative unit divided, is calculated through formula 1 , and the maximum value is taken as the $A Q I$ of the city. The calculation formula is as follows (2):

$$
A Q I=\max \left\{I A Q I_{1}, I A Q I_{2}, \cdots, I A Q I_{n}\right\}
$$

IAQI is the sub index of air quality, $n$ is the number of pollutant items.

\subsection{Pearson Correlation Coefficient}

Pearson correlation coefficient PCC is a statistical method used to quantitatively measure the correlation between variables. Its value is between -1 and 1 . The greater the absolute value, the stronger the correlation. This experiment will use Pearson correlation coefficient to measure the linear correlation between different air pollutant concentrations.

Firstly, the correlation between two random variables is calculated through covariance, as shown in equation (3):

$$
\operatorname{cov}(X, Y)=\frac{\sum_{i}^{n}\left(X_{i}-\bar{X}\right)\left(Y_{i}-\bar{Y}\right)}{n-1}
$$

Pearson correlation coefficient is calculated by equation (4):

$$
\rho(X, Y)=\frac{\operatorname{cov}(X, Y)}{\sigma_{X} \sigma_{Y}}
$$

$\operatorname{cov}(X, Y)$ is the covariance between two variables, $\sigma_{X}$ and $\sigma_{Y}$ are standard deviation of variables.

\section{Results and Analysis}

In the process of urban air pollution, it is mainly affected by the following two factors: first, the distribution and emission of various pollution sources and the influence of pollutants on each other; Second, the impact of meteorological conditions on the diffusion, dilution and migration of air pollutants.

\subsection{The Correlation Analysis between Pollutants and Meteorological Factors}

Table 1: Correlation coefficient between pollutants and meteorological factors in Sichuan Province in March 2018

\begin{tabular}{|c|c|c|c|c|c|c|}
\hline Type & PM2.5 & $\mathbf{P M}_{\mathbf{1 0}}$ & $\mathbf{S O}_{\mathbf{2}}$ & $\mathbf{N O}_{\mathbf{2}}$ & $\mathbf{C O}$ & $\mathbf{O}_{\mathbf{3}}$ \\
\hline Temp & -0.46 & -0.45 & -0.39 & -0.57 & -0.65 & 0.51 \\
\hline RH & 0.23 & 0.29 & -0.04 & -0.37 & 0.44 & 0.45 \\
\hline Pressure & 0.31 & 0.29 & 0.46 & -0.29 & 0.35 & -0.12 \\
\hline Windy & -0.48 & -0.51 & -0.34 & -0.65 & 0.06 & -0.60 \\
\hline
\end{tabular}

The Pearson correlation coefficient between pollutants and meteorological factors in Sichuan Province in March 2018 is counted, and the radar chart is used for big data visual display, as shown in Figure 1. Where $r=-1$ is the center of the circle, indicating complete negative correlation, and $\mathrm{R}=1$ is the corresponding point on the arc, indicating complete positive correlation.

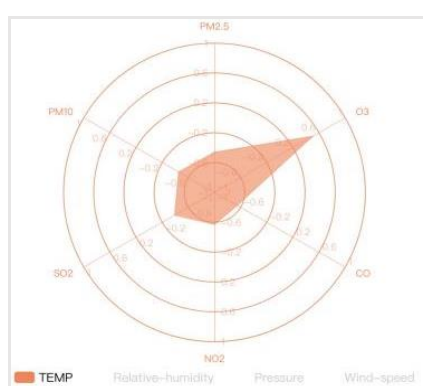

(a) Correlation between temperature and pollutants

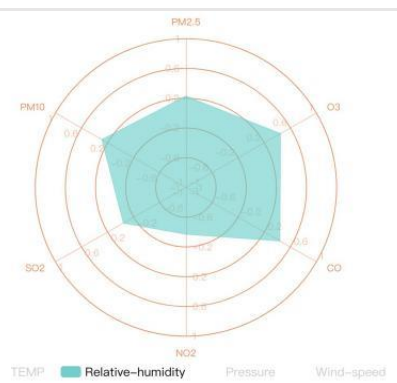

(b) Correlation between relative

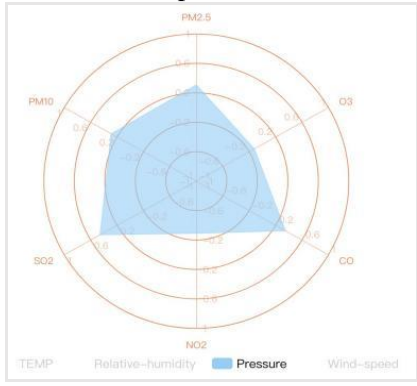

(c) Correlation between pressure and pollutants humidity and pollutants

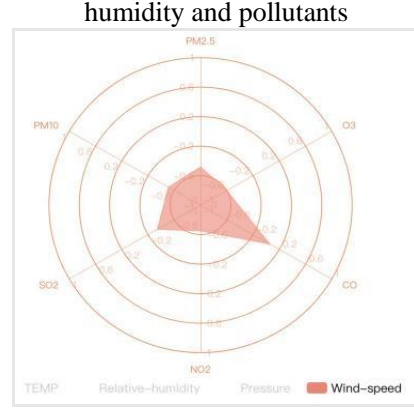

(d) Correlation between wind speed and pollutants
Figure 1: Visualization of correlation between pollutants and meteorological factors (radar chart)

According to the data analysis in Table 1 and figure 1, the meteorological factors (temperature, humidity, pressure and wind speed) affecting air pollution in Sichuan Province in March 2018 have different degrees of correlation with six conventional pollutants.

As shown in Figure 1(a), temperature has an obvious impact on six pollutants, and there is a positive linear relationship between ozone concentration in the atmosphere and temperature. The temperature is negatively correlated with other pollutants, because the temperature is conducive to the vertical flow of ground hot air, which is also conducive to the diffusion of pollutants. Because the atmospheric diffusion conditions are relatively good in summer and there are more rainfall, it can remove air pollutants to a certain extent. In winter, due to the high frequency of adverse meteorological conditions such as temperature inversion, it is difficult to diffuse pollutants, which is also a reason why the air quality in 
summer is better than that in winter. As can be seen from Figure 1 (b), the influence of relative humidity on air pollutants is small, which is positively correlated with PM2.5, $\mathrm{PM} 10, \mathrm{CO}$ and $\mathrm{O}_{3}$, and slightly negatively correlated with $\mathrm{SO}_{2}$ and $\mathrm{NO}_{2}$, because the increase of humidity will dilute the concentration of pollutants. As can be seen from Figure 1 (c), the atmospheric pressure shows a weak positive correlation with the concentration of other pollutants. As can be seen from Figure 1 (d), wind speed has a negative correlation with most pollutants and a weak positive correlation with Co. it can be seen that wind speed has a close relationship with the diffusion of air pollution.

\subsection{Analysis of Coastal Areas in Northern China}

According to the division standard of the National Bureau of statistics, China is divided into economic regions and geographical regions. The economic region is divided into the 8 regions.

Next, four areas in the northern coastal area are deeply studied. Visualize the evolution of air pollution in Beijing, Tianjin, Hebei and Shandong from 2013 to 2018, as shown in Figure 2.

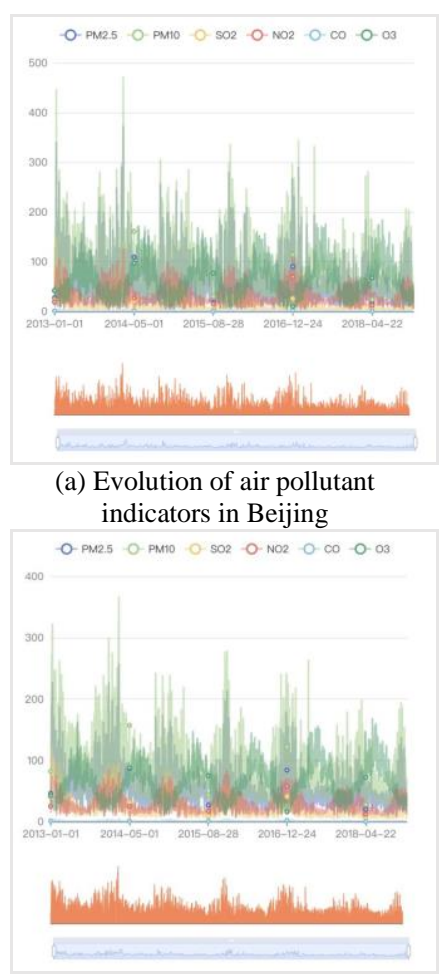

(c) Evolution of air pollutant indicators in Hebei Province

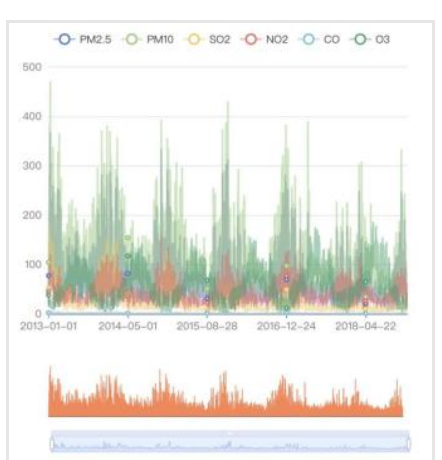

(b) Evolution of air pollutant indicators in Tianjin - - PM2.5 - - PM10 - $-502-0-$ - NO2 - - CO - - - 03 500

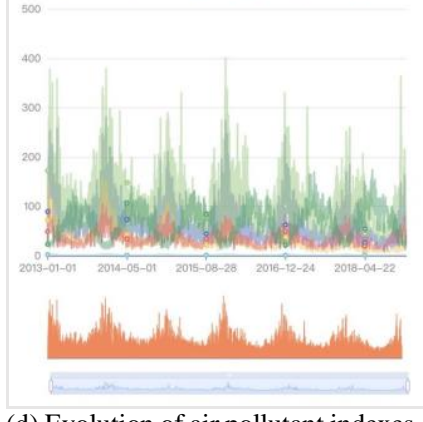

(d) Evolution of air pollutant indexes in Shandong Province
Figure 2: Visualization of air pollutant index evolution (stack diagram)

It can be seen from Figure 2 that from the perspective of time, the air pollution in the northern coastal areas presents obvious seasonal characteristics. The serious air pollution is mainly concentrated in autumn and winter, especially in winter. The air pollution is mainly coal smoke pollution, and the main pollutants are PM2.5 and PM10. In December and January of each year, there are the effects of heating period and dust storm, aggravation of air pollution and obvious problems of $\mathrm{SO}_{2}$ pollution. With the increase of years, the pollution situation in various regions shows a trend of steady improvement.

\section{Conclusion}

1) From the perspective of breadth, this paper calculates the Pearson correlation coefficient between $\mathrm{PM}_{2.5}, \mathrm{PM}_{10}, \mathrm{SO}_{2}$, $\mathrm{NO}_{2}, \mathrm{CO}, \mathrm{O}_{3}$ and meteorological factors (TEMP, RH, PSFC, windy) according to the monthly granularity of Chinese cities with 6-year full-scale data, and comes to the conclusion that meteorological factors have a great impact on Pollutants. Among them, temperature has a negative correlation with pollutants, air pressure has a positive correlation, and humidity has no significant correlation, there is an obvious negative correlation between wind speed and six pollutants.

2) Geographical, economic and climatic factors have a great impact on air pollution. The air pollution in spring and summer is significantly better than that in autumn and winter. The peak air pollution concentration is mainly concentrated in January and December. The source and diffusion of air pollutants is a comprehensive and complex process, which is the result of multiple factors. In terms of air pollution control, the emission and diffusion of pollution sources should be controlled.

\section{Acknowledgments}

We would like to thank the sponsors of the College Students' innovation and entrepreneurship training program of Sichuan University of Science and Engineering (No.cx2021093).

\section{References}

[1] Chai Fahe. Review and Prospect of air pollution control in China [J]. Environment and Sustainable Development, 2020, 45(03): 5-15.

[2] Zhang Jieqiong, Wang Yaqian, Gao Shuang, et al. KZ filtering study on the relationship between meteorological elements and air pollution at different time scales [J]. China Environmental Science, 2018, 38(10): 64-74.

[3] Tang Jiaxiang, Fu Chuanbo, Yang Renyong, et al. Evolution characteristics of PM2.5 in Haikou City and its relationship with meteorological factors $[\mathrm{J}]$. Environmental Pollution and Prevention, 2018, 40(4): 445-449.

[4] Tan Lina. Relationship between the distribution of air pollutants and meteorological factors[J]. Environmental Science and Management, 2021, 46(03): 64-68.

[5] Fang Xiaoting, Duan Huabo, Hu Mingwei. Seasonal difference analysis and prediction model comparison of the impact of meteorological factors on air pollutants --a case study of Shenzhen [J]. Environmental Pollution and Prevention and Control, 2019, 41(5): 541-546.

[6] Yang Liu, Wang Shigong, Zhang Ying. Variation characteristics of air pollutants in Chengdu in recent three years and the impact of precipitation on them $[\mathrm{J}]$. Journal of Lanzhou University (Natural Science Edition), 2018, 54(6): 731-738

[7] Liu Jian, Liu yinkun. Study on the correlation between meteorological factors and pollutants based on Pearson coefficient [J]. Journal of North China University of Science and Technology, 2019, 16(04): 93-97. 
[8] Wang Geng, Liu Yijiang, Wang Lijing, et al. Correlation analysis of air pollution characteristics and meteorological factors in Zhangqiu District [J]. Journal of Liaoning Normal University (Natural Science Edition), 2018, 41(04): 516-522.

[9] Liu Qing, Li Dian, Wang Liwei, et al. Study on correlation modeling between air pollutant concentration variation characteristics and meteorological factors [J]. Environmental Science and Management, 2021, 46(04): 136-140.

[10] Liu Zihao, Huang Jianwu, Kong Deya, et al. Temporal and spatial variation of air pollution and its correlation with meteorological factors in Hefei [J]. Environmental Science and Management, 2019, 44(02): 43-48.

[11] Li Bo, Chen Wei, Fu Qiang, et al. Canonical correlation analysis of ambient air pollutants and meteorological elements in Heilongjiang Province [J]. Environmental Monitoring in China, 2021, 37(03): 103-108.

[12] Lei K, Xiao T, et al. A 6-year-long (2013-2018) high-resolution air quality reanalysis dataset in China based on the assimilation of surface observations from CNEMC, Earth Syst[J]. Sci. Data, 2021, 13: 529-570. 